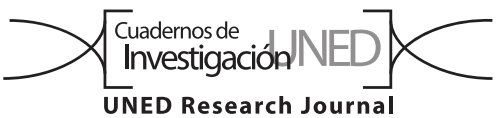

\title{
Nuevo modelo para tutorías de Física y su efecto en el rendimiento estudiantil en una universidad a distancia de Costa Rica
}

\author{
Diana Herrero-Villarreal, Yency Calderón Badilla \& Carmen Andrés Jiménez \\ Universidad Estatal a Distancia (UNED), 474-2050 Mercedes de Montes de Oca, San José- Costa Rica; dvillarreal@uned.ac.cr; \\ ycalderon@uned.ac.cr; candres@uned.ac.cr
}

Recibido 06-IX-2017 • Corregido 28-XI-2017 • Aceptado 07-XII-2017

\begin{abstract}
New model for Physics tutorials and its effect on student performance at a distance university in Costa Rica. Student self-regulation, previous preparation of tutors and collaborative work are key factors in distance education. We tested a new tutoring design in the Physics for Science Teaching III course of the Distance State University of Costa Rica (UNED). A survey indicated that teachers must focus on student motivation; class environment -which influences student focus; and the fact that tutoring is a good tool for diagnostic and formative evaluation. Performance improved with the new model. We recommend strategies to improve self-assurance and performance in written tests, collaborative work and individual attention.
\end{abstract}

Key words: Tutoring, distance education, academic performance, motivation.
RESUMEN: La autorregulación del estudiante, la preparación previa de tutores y el trabajo colaborativo son factores clave en la educación a distancia. Probamos un nuevo diseño de tutoría en el curso de Física III para la Enseñanza de la Ciencia de la Universidad Estatal a Distancia de Costa Rica (UNED). Una encuesta indicó que los profesores deben enfocarse en la motivación del estudiante; ambiente de la clase -que influye en la concentración del estudiante; y el hecho de que la tutoría es una buena herramienta para el diagnóstico y la evaluación formativa. El rendimiento mejoró con el nuevo modelo. Recomendamos estrategias para mejorar la seguridad y el rendimiento del estudiante en pruebas escritas, trabajo colaborativo y atención individual.

Palabras clave: Tutorías, educación a distancia, rendimiento académico, tutores, motivación.
Para que una tutoría sea realmente exitosa, la cátedra de Física de la Escuela de Ciencias Exactas y Naturales (ECEN), determinó aspectos importantes como lo son: la autorregulación del estudiantado, el rol del personal docente en su preparación y el fomento del trabajo colaborativo en el estudiantado (Calderón, Herrero \& Andrés, 2017).

Antes de entrar en estos tres temas, es importante definir lo que se entiende por tutoría. La evolución de la educación a distancia ha dado lugar a diferentes tipos o modalidades de tutorías, las cuales se han originado atendiendo a la naturaleza y filosofía de la entidad educativa, a los recursos disponibles, al número y ámbito de la población beneficiaria y a los objetivos a conseguir.

De acuerdo con Rodríguez (2014), en términos generales podemos establecer dos tipos de tutorías utilizadas por las instituciones de enseñanzas y aprendizajes abiertos y a distancia: la tutoría presencial y la tutoría a distancia. La tutoría presencial puede llevarse a cabo individualmente, en grupo, en la institución, en centros regionales e itinerantemente (a través de tutores viajeros), mientras que la tutoría a distancia, que es el caso que le atañe a la UNED de Costa Rica, ha adquirido una serie de matices en los que se puede aplicar, ya sea de forma esporádica en un aula, como se ha hecho tradicionalmente desde inicios de la universidad, hasta mediante sesiones sincrónicas en salas virtuales como Scopia o WizIQ o la plataforma virtual Moodle, mediante sesiones asincrónicas.

Es así como a través de una tutoría, se puede fomentar la autorregulación y el trabajo colaborativo, mediado por el rol del personal docente.

Con respecto a la autorregulación de los aprendizajes, el Modelo Pedagógico de la UNED (2004), fomenta esta práctica como pilar fundamental de la educación a distancia. Según Rodríguez (2014), para ello, el personal 
docente debe fomentar en todo momento la colaboración y la participación en actividades y prácticas diversas, dado que el aprendizaje es un proceso que necesita de colaboración, diálogo e interacción entre todos los actores y otros recursos dentro del contexto social y cultural propio del discente. Una de las maneras en que la tutoría en la UNED puede fomentar la autorregulación y por lo tanto un mejor rendimiento, es mediante el uso de herramientas tecnológicas que complementen esta forma de enseñar-aprender.

Con respecto al rol que el personal docente debe tener, es importante considerar que consiste en un apoyo temporal que brinda, en un espacio real o virtual, para que el estudiantado desarrolle todo su potencial en su proceso de aprendizaje. En otras palabras, este rol empieza por medio de una preparación concienzuda en el dominio de la materia que debe impartir y a partir de la cual debe planificar un ambiente de aprendizaje óptimo donde poder aclarar todas las dudas que surjan en el estudiantado, así como orientarles en su proceso de aprendizaje. Debe tener la capacidad pedagógica de compartir los contenidos de manera clara y suficiente con los estudiantados, despertando en ellos el interés por cumplir los objetivos de aprendizaje.

Sobre las oportunidades que dan las tutorías, destaca la de generar estrategias de evaluación de los aprendizajes de carácter diagnóstico y formativo. En este sentido el tutor puede determinar en la tutoría, a partir de técnicas como la observación y la entrevista aspectos socio-afectivos-geográficos, entre otros del estudiantado, pero, también identificar el grado de conocimiento que posee acerca de los contenidos de la asignatura mediante la aplicación de diversos instrumentos y actividades como cuestionarios, debates, preguntas generadoras, entre otros.

El implementar la evaluación formativa en la tutoría brinda la posibilidad de interiorizar el concepto de mejora en el estudiantado, entendiendo que el proceso de aprendizaje siempre estará sujeto a las capacidades que posean los estudiantados para autorregularlo y a la disposición que tengan para ver en cada "error" la oportunidad de corregir y mejorar. Como afirma Fasce (2009), "el eje central de la evaluación formativa es proveer retroalimentación al estudiantado sobre su progreso y sus debilidades, tendiendo a favorecer sus habilidades de aprendizaje", por lo tanto, podría aplicarse exámenes cortos, trabajos grupales, resolución de ejercicios, entre otros que le permitan al estudiantado valorar su avance $y$ al docente retroalimentarlo.

Una vez que el tutor o la tutora logró empoderarse de su rol en un sistema de enseñanza a distancia, el siguiente paso es fomentar el trabajo colaborativo dentro de las estrategias metodológicas utilizadas en la tutoría. Según Alvarado y Romero (2007), desde la perspectiva de comunidades de aprendizaje, la tutoría se caracteriza por un acompañamiento cuya interacción es activa y dinámica por sus prácticas de colaboración y por la responsabilidad de sus participantes en cuanto a la construcción de conocimientos y la comprensión propia de la materia.

La presente investigación tiene como objetivo realizar un análisis de una propuesta para la tutoría presencial en la asignatura Física III para la Enseñanza de las Ciencias, de la carrera Enseñanza de las Ciencias Naturales, en dos centros universitarios distintos a los que asisten estudiantados de diversas partes de Costa Rica: San José y San Isidro.

\section{METODOLOGÍA}

Para determinar la eficacia de una nueva estrategia para impartir tutorías presenciales y su efecto en la autorregulación del estudiantado, el rol del docente y el fomento del trabajo colaborativo; se aplicó en un grupo de estudiantes de un cuatrimestre y asignatura específicas. Posteriormente, para evaluar la eficacia de esta propuesta, se realizaron dos tipos de análisis. El primero fue la aplicación de una encuesta al estudiantado y al personal docente que estuvo involucrado en esta nueva manera de trabajar y el segundo fue un análisis estadístico para comparar ente las calificaciones obtenidas en el grupo que recibió la tutoría de forma tradicional y el que la recibió con esta nueva metodología.

Aplicación de la nueva metodología para impartir tutorías: Se aplicó la propuesta innovadora diseñada en Calderón, Herrero y Andrés (2017), para el desarrollo de tutorías presenciales, a la asignatura Física III para la Enseñanza de las Ciencias en el tercer cuatrimestre 2016 con dos tutores diferentes.

La propuesta se puede observar en el apéndice digital 1. Entre los contenidos más importantes desarrollados en esta propuesta se encuentran la experiencia previa de la materia, tanto por parte del estudiantado como del personal docente y la realización de un quiz antes de la tutoría, que permita y obligue el repaso de los temas que se verán en clase.

La aplicación de la propuesta, a manera de plan piloto, permitió realizar una validez de resultados con el propósito de dar insumos a la cátedra acerca de la pertinencia e importancia de aplicar esta nueva metodología. 
Elaboración de encuestas para personal docente y estudiantado: Para determinar la percepción que tanto el personal docente como el estudiantado tuvieron de la implementación de esta nueva experiencia, se procedió a elaborar dos instrumentos de medición. Los instrumentos utilizados se pueden observar en los apéndices digitales 2 y 3.

Ambas encuestas pasaron por un proceso de validación que se describe en Calderón, Herrero y Andrés (2017). Sin embargo, decidimos incorporar la posibilidad de responder entre un rango de 0 a 5, en vez de obtener respuestas de "sí" o "no", ya que esa fue una limitante que se encontró la última vez que se aplicaron.

En la encuesta para el personal docente se incorporaron los siguientes temas:

- Anotar el esquema de distribución de tiempo en una tutoría.

- Anotar las estrategias de trabajo utilizadas durante la tutoría.

- Si implementó el plan piloto de tutorías impulsado por la cátedra, anotar las observaciones que le parezcan pertinentes sobre el mismo.

Análisis del rendimiento académico: Se calculó el porcentaje de aprobación del estudiantado desde el tercer cuatrimestre del año 2014 hasta el año 2016, obteniendo la cantidad de estudiantes que aprobaron y el total de estudiantes para cada año.

Análisis estadístico de la información: Se realizaron dos pruebas estadísticas a partir de la información que los resultados arrojaron. La primera fue una prueba de U de Mann-Whitney para determinar si el haber diferente personal docente en cada grupo estudiado, generó diferencias significativas en las respuestas dadas por el estudiantado. La segunda fue una prueba de comparación de proporciones entre parejas, para determinar si hubo diferencias significativas y si se pueden atribuir de alguna manera al cambio realizado en la metodología de las tutorías.

\section{RESULTADOS}

En esta experiencia se encontró que el nuevo modelo de tutorías fue posible implementarlo con oportunidades de mejora para la cátedra. El estudiantado expresó su motivación hacia este nuevo modelo dado que le ayuda en las actividades evaluativas de la asignatura, pero aún es necesario lograr que el sistema incida en una mejora de su rendimiento académico. El personal docente manifestó que el modelo de tiempos debe ser menos rígido pero que en general es muy útil para lograr una tutoría más participativa y que involucre diferentes elementos que promueven el aprendizaje.

Aplicación de la propuesta innovadora para impartir tutorías: Se puede constatar que, aunque se acordó un esquema de distribución de tiempos para la tutoría bastante detallada, cada docente adaptó el esquema a sus necesidades específicas en el aula. Aunque ambos mantuvieron la esencia de la propuesta que consistía en incorporar trabajo práctico del estudiantado durante la tutoría y reducir la cantidad de tiempo dedicada a la exposición magistral de los contenidos de la asignatura.

Percepción del personal docente y el estudiantado con respecto a la nueva metodología: A continuación, se presentan los principales resultados obtenidos a partir de la implementación de la propuesta innovadora para impartir tutorías presenciales en la asignatura Física III.

Con respecto a las respuestas obtenidas en la encuesta aplicada al estudiantado para determinar su percepción sobre la nueva estrategia utilizada en las tutorías, se encontró que la percepción general del estudiantado con respecto a este nuevo modelo es positiva en cuanto a motivación, aprovechamiento, giras, pruebas cortas, laboratorios; sin embargo, en algunos casos se encontró que no fue tan positiva en mejorar su rendimiento ni su aprendizaje en las pruebas escritas.

En el apéndice digital 4 se puede observar la prueba de $U$ de Mann-Whitney y W de Wilcoxon utilizada para determinar diferencias entre los dos grupos, cada uno con diferente tutor. En esta prueba se encontró una diferencia significativa entre el nivel de orientación que el personal docente está proporcionando.

En el Cuadro 1 se muestran los comentarios que el estudiantado dio sobre su percepción acerca de la nueva experiencia. Se puede constatar en el Cuadro 1, que el trabajo docente, así como la evaluación son calificados positivamente, no así el entorno de la tutoría. La infraestructura parece tener serias deficiencias en cuanto a ruido, iluminación y ventilación. Las sugerencias van en el ámbito de la metodología de las tutorías, el entorno virtual y el libro de texto.

A continuación, se muestran los resultados más importantes de la encuesta aplicada al personal docente, los cuales son diferentes por partes de las dos personas encargadas de aplicarlo. Por un lado, el tutor de San Isidro, quien dedicó 30 minutos por sesión a atención de consultas sobre pruebas cortas en línea, luego 2 horas a 
explicación de la teoría, atención de consultas sobre la teoría y resolución de ejercicios. Por último, 30 minutos de trabajo grupal y exposición de los problemas asignados. Por otro lado, la tutora de San José utilizó los primeros 10 minutos para realizar un saludo y discusión sobre situaciones del curso (asuntos administrativos). Luego, 20 minutos para explicar los conceptos más relevantes, 20 minutos para resolver uno o dos problemas relevantes, 10 minutos para que estudiantados resolviesen ejercicios de forma individual o grupal y por último discusión de los ejercicios.

Los aspectos positivos que el personal docente encontró de la aplicación de esta nueva experiencia fueron la mayor participación y permanencia del estudiantado, menos temor a la hora de preguntar dudas y mejores resultados en los exámenes.

Como parte de los temas pendientes de mejorar, ambos tutores indicaron que durante la tutoría surgen imprevistos que a veces no permiten cumplir con los tiempos establecidos, así como el hecho de que el estudiantado debe llevar la materia leía antes de ir a la tutoría.

Las estrategias de trabajo más utilizadas por ambos tutores fueron: exposición magistral y trabajo en grupos. Aunque se puede constatar según lo indicado por la tutora de San José, que también se aplicó trabajo individual.

\section{CUADRO 1}

Comentarios hechos por el estudiantado en la encuesta aplicada

\begin{tabular}{cl} 
Aspecto & \multicolumn{1}{c}{ Resultados } \\
Empatía hacia la persona que imparte la tutoría & $\begin{array}{l}\text { Me gusta la forma en que la tutora imparte la tutoría } \\
\text { Estoy satisfecho con el desempeño de la tutora. } \\
\text { Mantener a la tutora que explica de manera excelente. }\end{array}$
\end{tabular}

Entorno en el que se brinda la tutoría

Los alrededores del aula son bulliciosas.

La iluminación del aula no es adecuada.

El aula está junto al parqueo de autos y provoca distracción. Hay poca ventilación en el aula.

Instrumentos de evaluación

La cantidad de instrumentos es apropiada.

Las fechas para aplicar las pruebas cortas son adecuadas.

Podrían aplicarse pruebas cortas después de cada tutoría, de manera presencial o virtual.

Otros apoyos para el proceso de aprendizaje

Podrían darse al menos 2 tutorías más.

Realizar ejercicios en la tutoría que permitan practicar para el examen. Implementar en el entorno virtual materiales de apoyo complementario como: resúmenes, lecturas, prácticas, entre otros.

Actualizar el libro de texto.

Rendimiento académico: El rendimiento académico del estudiantado desde el año 2014 hasta el 2016; periodo en el cual se impartían las tutorías de forma tradicional y se implementó la mejora de esta experiencia.

El porcentaje de aprobación en los cuatrimestres en los que no se aplicó la nueva metodología de trabajo fue menor al porcentaje obtenido durante el cuatrimestre en el que sí se utilizó. Es decir, hubo mayor respuesta de parte de los estudiantes que en los cuatrimestres anteriores. Pero no se obtuvieron diferencias significativas entre años 2014y $2016(p=0,03)$ y 2015 y 2016 $(p=0,219)$ (Fig. 1).

\section{DISCUSIÓN}

Esta experiencia arrojó resultados a la luz de los tres grandes temas que se desarrollaron alrededor de ella: la

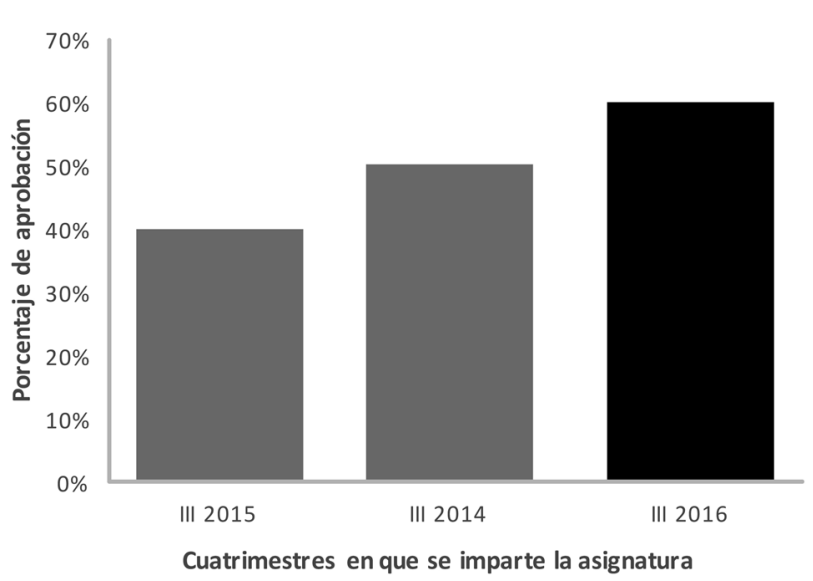

Fig. 1. Porcentaje de aprobación en la asignatura Física III durante el tercer cuatrimestre por tres años seguidos: 20142016. Se resalta en negro el cuatrimestre en el cual se aplicaron las mejoras. 
autorregulación, el rol del personal docente y el fomento del trabajo colaborativo. Analizaremos cada uno de ellos.

El poder constatar un rendimiento académico mejor en el cuatrimestre analizado en la presente experiencia respecto a los dos anteriores, aunque no significativo, puede sugerir que las mejoras aplicadas a la tutoría, de alguna manera influenciaron en la motivación del estudiantado para autorregularse y obtener mejores calificaciones, lo cual también se constata con las respuestas que ellos dieron en la encuesta.

No obstante, no podría afirmarse aún que el mejor rendimiento académico se debe únicamente al factor de la metodología de la tutoría. De hecho, de acuerdo a Alvarado y Romero (2007), "el rendimiento escolar de los alumnos, está determinado por sus antecedentes familiares, características individuales, antecedentes educativos y los compromisos para alcanzar sus metas. Así, tanto el sistema escolar como el alumno, son retroalimentados por el sistema social" (p.4).

En los resultados obtenidos en esta investigación, parece haber un efecto a nivel motivacional y de ambiente de confianza en el aula puesto que la docente indica que notó "menos temor a la hora de preguntar dudas", lo cual se traduce en una mayor autorregulación. De acuerdo a Pagano (2007), aunado al cambio en la estrategia metodológica a utilizar, el tutor, o la tutora, debe tener atributos en las dimensiones didácticas, técnicas y psicoactivas.

Ahora bien, la percepción del estudiantado también arroja oportunidades de mejora con respecto a su autorregulación, por ejemplo, el hecho de que una parte no encontrara tan beneficiosa la tutoría para poder prepararse para las pruebas escritas, lo cual, implica que se debe modificar el enfoque que se le da a este tema en las tutorías para que sea tangible su utilidad. Al respecto, una experiencia realizada por Stenlund, Eklöf y Lyrén (2017), en donde se compararon dos grupos de estudiantes, unos con notas altas y otros con notas bajas en pruebas escritas, encontró que los que sacaron notas altas, tenían estrategias exitosas de aprendizaje que los llevaron a estos resultados, mientras que los otros no.

Para mitigar la situación mencionada anteriormente, se recomienda utilizar la estrategia sugerida por Jury, Smeding, Stephens, Nelson, Aelenei y Darnon (2017), en donde a partir de una experiencia sobre las razones por las que hay bajo rendimiento en pruebas escritas, se recomendaron una serie de estrategias relacionadas con la enseñanza que generen alta autoestima en el estudiantado, enfatizando la necesidad de más ayuda y trabajar con metas a corto plazo que se puedan cumplir.

Seguidamente, con respecto al rol del docente en el nuevo modelo de tutorías aplicado, si bien en los espacios de tutoría se promueven el acercamiento simultáneo de tiempo y espacio, la figura del docente en la metodología de educación a distancia tiene un contexto de guía. De acuerdo con Zelaya (2007), un tutor debe lograr la motivación del aprendizaje, que presta su apoyo en caso necesario; de esta manera, el seguimiento y la adecuada comunicación entre estudiantado- personal docente, han de ser oportunas tanto en las tutorías como en otros espacios, sincrónicos o asincrónicos, que sean parte del desarrollo de la asignatura. Sin embargo, los resultados sugieren cómo el modelo a distancia es una cultura en constante desarrollo y se recomienda, por lo tanto, buscar un balance entre la guía brindada y el acompañamiento más de cerca en los casos en los que se requiera, hasta lograr la formación de esta cultura.

Al encontrarse diferencias significativas entre los dos tutores que aplicaron el nuevo modelo respecto a su función orientadora en su proceso de aprendizaje, indica que hubo mayor satisfacción en el estudiantado liderado por uno de los tutores; por lo tanto, se recomienda trabajar este punto con el tutor que fue calificado de forma menos favorable.

Adicionalmente, cabe destacar que la población estudiantil, no solamente valora que la persona que imparta la tutoría muestre dominio del contenido o explique de manera adecuada; sino que, para el estudiantado es necesario que la tutoría se imparta en espacios físicamente adecuados. Al existir distracciones auditivas y visuales en el exterior de la clase se pueden producir limitaciones en la captación del mensaje, interrumpiendo los procesos mentales básicos del estudiantado, tales como: atención y concentración. Tal como lo afirma López (s.f.):

"El ruido repentino puede producir distracciones que reducirán el rendimiento en muchos tipos de trabajos, especialmente en aquellos que exijan un cierto nivel de concentración. En estos casos se afectará la realización de la tarea, apareciendo errores y disminuyendo la calidad y cantidad del producto de la misma" (p.9).

Finalmente, la experiencia arroja resultados positivos y con oportunidad de mejora respecto a la aplicación del trabajo colaborativo. Diferentes experiencias coinciden con la hipótesis de esta experiencia, con respecto a la aplicación de modelos en donde se propicie el trabajo colaborativo y el aprendizaje. Según un estudio realizado por Maldonado, Bencomo, Franco, Santos, Subía y Alarcón (2017), la estrategia pedagógica denominada clase invertida, es una modalidad que está tomando fuerza principalmente porque la construcción del conocimiento de forma colaborativa es beneficioso para el aprendizaje. Asimismo, García, Domínguez y Spitcich (2017), en una investigación realizada también 
en asignaturas de Física a nivel superior, encontraron que se puede afirmar que, el trabajo colaborativo proporciona un nivel de innovación importante en los procesos de enseñanza aprendizaje.

Se concluye que el nuevo modelo de tutoría aplicado a la asignatura Física III para la Enseñanza de las Ciencias tuvo una aceptación mayormente positiva entre los estudiantados y tutores. Esto se combina con un mejor rendimiento académico en el período en experiencia de forma tal que se valora la iniciativa como beneficiosa para el estudiantado.

Asimismo, se identificaron oportunidades de mejora para ser implementadas durante el próximo cuatrimestre que se imparta, específicamente relacionadas con que se debe enfatizar en el éxito en las pruebas escritas y el rendimiento de los estudiantados durante la tutoría.

A pesar de haber encontrado diferencias significativas como se mencionó anteriormente, una de las deficiencias metodológicas de esta experiencia, es que no se trabajó con un grupo control, para lo cual se recomienda se pruebe esta metodología en futuros cuatrimestres incluyendo un grupo en el que no se la aplique la mejora y así poder reforzar el hecho de que el rendimiento sea mayor al de otros cuatrimestres efectivamente se deba a la nueva metodología utilizada.

Se deberá trabajar en que cada docente imparta la tutoría, aún bajo sus términos, siguiendo una metodología que enfatice el trabajo colaborativo y las funciones tanto académicas como orientadoras y motivacionales de la tutoría.

\section{AGRADECIMIENTOS}

Se agradece a los estudiantes y tutores de la Cátedra de Física por su valiosa ayuda en esta experiencia, así como a Ligia Bermúdez de la UNED por su colaboración en el análisis estadístico.

\section{REFERENCIAS}

Alvarado, V., \& Romero, R. (2007). La tutoría en la educación a distancia. Revista de Planeación y Evaluación Educativa, 38. Recuperado de https://www.researchgate.net/publication/278963520_Tutoria_en_educacion_a_distancia

Calderón, Y., Herrero, D., \& Andrés, C. (2017). Fortalezas y debilidades de las tutorías en educación universitaria a distancia: resultados de talleres con tutores de ciencias exactas y naturales. UNED Research Journal, 9(1), 179-184.

Fasce, E. (2009). Evaluación formativa. Revista Educación Cienc Salud, 6(1), 8-9. Recuperado de http://www2.udec.cl/ ofem/recs/anteriores/vol612009/esq61.pdf

García, D., Domínguez, A., \& Spitcich, S. (2017). Trabajo colaborativo para el desarrollo de prácticas innovadoras en la enseñanza de la física universitaria con el uso de tecnologías. Revista de Enseñanza de la Física, 29(1), 7-23.

Jury, M., Smeding, A., Stephens, N., Nelson, J., Aelenei, C., \& Darnon, C. (2017). The Experience of Low-SES Students in Higher Education: Psychological Barriers to Success and Interventions to Reduce Social-Class Inequality. Journal of Social Issues, 73(1), 23-41.

López, L (s.f). ¿Normalización de ruido en bibliotecas? Especialización en Ingeniería Ambiental Instituto Tecnológico de Ciudad Juarez. Recuperado de http:// www.semac.org.mx/archivos/5-17.pdf

Maldonado, F., Bencomo, O., Franco, J., Santos, M., Subía, J., \& Alarcón, L. V. (2017). La educación invertida. Un nuevo reto para la educación superior. Revista de la Facultad de Ciencias Médicas, 2017, 40-45.

Pagano, C. (2007). Los tutores en la educación a distancia. Un aporte teórico RUSC. Universities and Knowledge Society Journal, 4 (2), 1-11. Recuperado de http://www.redalyc. org/pdf/780/78011231005.pdf

Rodríguez, N. (2014). El tutor frente a la educación a distancia: concepciones, funciones y estrategias tutoriales. Revista de Cooperación, 5, 2308-1953. Recuperado de http:// www.revistadecooperacion.com/numero3/03-04.pdf

Stenlund, T., Eklöf, H., \& Lyrén, P. E. (2017). Group differences in test-taking behaviour: An example from a high-stakes testing program. Assessment in Education: Principles, Policy \& Practice, 24(1), 4-20.

Zelaya, J. (2007). Educación a Distancia: Una alternativa para los sistemas educativos. San José, Costa Rica: Colección IDER. 